\title{
MOMENTO DE ADICIÓN DEL GLICEROL SOBRE LA CALIDAD ESPERMÁTICA EN LA CRIOPRESERVACIÓN DE SEMEN CANINO
}

\author{
Timing of Glycerol Addition on Sperm Quality in Cryopreservation \\ of Canine Semen
}

Gino Carlotto R. ${ }^{1}$, Víctor Fernández A. ${ }^{2}$, Boris Lira M. ${ }^{3}$, Alexei Santiani A. ${ }^{1,4}$

\section{Resumen}

\begin{abstract}
El objetivo del presente trabajo fue evaluar el efecto del momento de adición del glicerol durante el proceso de enfriamiento sobre la calidad espermática del semen canino. Se utilizaron 15 eyaculados de 4 perros adultos. Cada muestra de semen fue dividida en alícuotas para someterlas a tres métodos de adición de glicerol (T1: al inicio; T2: al inicio y final; T3: al final de la curva de enfriamiento). El semen se envasó en pajillas de 0.5 $\mathrm{ml}$ y se congeló en nitrógeno líquido. Se evaluó la motilidad progresiva y la integridad funcional de membrana posdescongelamiento. No se encontró diferencia estadística entre los tres métodos de adición del glicerol, pudiéndose simplificar el proceso de criopreservación al añadir todo el glicerol al inicio de la curva de enfriamiento.
\end{abstract}

Palabras clave: semen, canino, criopreservación, glicerol

\section{Abstract}

The objective of the study was to evaluate the timing of adding glycerol during the cooling process on sperm quality of canine semen. Fifteen ejaculates from 4 mature dogs were used. Each sample was divided in aliquots and three methods of adding glycerol were used (T1: at the beginning; T2; at the beginning and at the end; T3: at the end of the cooling process). Semen samples were packed in $0.5 \mathrm{ml}$ straws and frozen in liquid nitrogen. The post-thawing progressive motility and the functional integrity of membrane were evaluated. None statistical difference was observed due to the three treatments, indicating that the process of cryopreservation can be simplified by adding all the glycerol at the beginning of the cooling curve.

Key words: canine, semen, cryopreservation, glycerol

\footnotetext{
${ }^{1}$ Laboratorio de Reproducción Animal, ${ }^{2}$ Clínica de Animales Menores, ${ }^{3}$ Laboratorio de Fisiología Animal, Facultad de Medicina Veterinaria, Universidad Nacional Mayor de San Marcos, Lima

${ }^{4}$ E-mail: asantiani@hotmail.com
} 
La crianza de perros de raza es una afición que está en constante crecimiento a nivel nacional e internacional. El producto de esta crianza son canes para exhibición, concursos, mascotas, o para trabajo (ganadería, policial, rescate, etc.). En este escenario, la criopreservación de semen y la inseminación artificial son técnicas reproductivas que brindan grandes ventajas, pues permiten conseguir un rápido progreso en el mejoramiento genético de las razas caninas, así como aparear animales ubicados en zonas geográficas distantes (Stornelli et al., 2001; Sánchez et al., 2006). Adicionalmente, la criopreservación del semen canino permite mantener reservas genéticas de animales de gran valor y de caninos silvestres que se encuentran en situación vulnerable o en peligro de extinción, ayudando de esta forma a su conservación (Linde-Forsberg, 1995).

Actualmente, en el Perú, la criopreservación de semen canino es de uso limitado o nulo, debido a que la congelación de semen canino es una técnica que requiere de equipamiento y personal especializado (Morton y Bruce, 1989; England, 1993). En este contexto, es necesario buscar una alternativa que simplifique la técnica de criopreservación, y reduzca posibles errores humanos durante su ejecución. Una posible acción podría estar en la simplificación del proceso de congelación, incluyendo la adición del crioprotector, junto con los demás componentes del dilutor, al inicio de la curva de enfriamiento (Silva et al., 2003).

Se han evaluado diversos métodos de adición de crioprotector con resultados prometedores (Peña et al., 1998; Yildtz et al., 2000; Nothling y Shuttleworth, 2005; Silva et al., 2006). El objetivo del presente estudio fue evaluar un método alterno en el proceso de criopreservación del semen canino.

\section{Lugar de Estudio y Animales}

El estudio se realizó entre los meses de febrero y noviembre de 2008, en el Laboratorio de Reproducción Animal de la Facultad de Medicina Veterinaria de la Universidad Nacional Mayor de San Marcos, Lima.

Se emplearon cuatro perros mestizos, con edades de 1 a 2.5 años, alojados en caniles, y mantenidos con alimento balanceado comercial y agaad libitum.

\section{Eyaculados y Dilutores}

Se trabajó con 15 eyaculados. El semen fue colectado dos veces por semana, mediante la técnica de manipulación digital (Kutzler, 2005).

En la dilución de los eyaculados se utilizó el dilutor descrito por Bateman (2001) y Hermansson y Forsberg (2006). Este dilutor consiste en $3.0250 \mathrm{~g}$ de TRIS (Amresco 0826), $1.4409 \mathrm{~g}$ de ácido cítrico anhidro (Sigma C0759) y $1.1260 \mathrm{~g}$ de fructosa (Mallinckridt 7756), disuelto en $100 \mathrm{ml}$ de agua destilada. Asimismo, 20\% de esta solución es reemplazada por yema de huevo (v/v).

\section{Tratamientos}

Se evaluó tres protocolos de adición de glicerol durante la curva de enfriamiento. Para esto, cada eyaculado se dividió en tres partes iguales para formar los siguientes grupos:

? T1 (Adición del glicerol al inicio de la curva de enfriamiento): El semen fresco $(1 \mathrm{ml})$ se diluyó en proporción 1:3 con el dilutor (glicerol 6.66\%), para llegar a una concentración final de glicerol del 5\%.

? T2 (Adición del glicerol al inicio y al final de la curva de enfriamiento): El semen fresco $(1 \mathrm{ml})$ se diluyó en propor- 
ción 1:1 con el dilutor (glicerol 6\%) para llegar a una concentración de 3\% de glicerol. Al final de la curva de enfriamiento se volvió a diluir agregando $2 \mathrm{ml}$ de dilutor (glicerol 7\%), para llegar a una concentración final de glicerol del 5\%.

? T3 (Adición del glicerol al final de la curva de enfriamiento): El semen fresco (1 ml) se diluyó en proporción 1:1 con el dilutor (sin glicerol). Al final de la curva de enfriamiento se volvió a diluir agregando $2 \mathrm{ml}$ de dilutor (glicerol 10\%), para llegar a una concentración final de glicerol del $5 \%$.

\section{Evaluación de Semen}

Se trabajó con un eyaculado por vez en cada ensayo. El semen se colectó en el laboratorio y se evaluó el volumen, motilidad progresiva, concentración espermática e integridad funcional de membrana. Se procesaron únicamente aquellas muestras con un volumen mínimo de $3 \mathrm{ml}$ y motilidad progresiva ?85\%.

La motilidad progresiva se evaluó colocando 20 ?1 de semen en una lámina portaobjetos y observando 10 campos mediante microscopía óptica a 400X, expresando el resultado en porcentaje. Se consideró como espermatozoides con motilidad progresiva aquellos que presentan un desplazamiento continuo hacia delante. La concentración espermática se midió con la cámara de Neubauer, y el resultado se expresó en millones de espermatozoides/mL (Ax et al., 2000).

La integridad funcional de membrana se evaluó mediante el test hiposmótico (HOS) descrito por Jeyendran et al., (1984) y modificado por Sánchez et al. (2002) para espermatozoides caninos. Se consideró como espermatozoides con membrana funcional aquellos espermatozoides que presentaron la cola hinchada o deformada en respuesta a la gradiente osmótica. Los resultados se expresaron en porcentaje de espermatozoides reaccionantes al test $\mathrm{HOS}(\mathrm{HOS}+)$.

\section{Criopreservación del Semen}

La criopreservación del semen se realizó en el siguiente orden: curva de enfriamiento, estabilización y congelación. La curva de enfriamiento se realizó colocando los tubos de los tres tratamientos en un recipiente con agua a $35^{\circ} \mathrm{C}$, que fue colocado en refrigeración por 60 min hasta conseguir un descenso gradual de la temperatura a $5^{\circ} \mathrm{C}$. Luego, se adicionó la segunda fracción de dilutor (temperada a $5{ }^{\circ} \mathrm{C}$ ) a los grupos 2 y 3 . Se mantuvo el semen de los 3 grupos por 15 min a $5{ }^{\circ} \mathrm{C}$ para la estabilización de los espermatozoides con el glicerol. Inmediatamente después se envasaron 2 pajillas de 0.5 $\mathrm{ml}$ por tratamiento. Finalmente, la congelación de las pajillas se hizo mediante exposición a vapores de nitrógeno por $15 \mathrm{~min}$ hasta alcanzar $-20{ }^{\circ} \mathrm{C}$, donde se sumergieron en nitrógeno liquido para su almacenamiento.

\section{Evaluación Posdescongelamiento}

El descongelamiento se realizó a las 24 horas de la criopreservación, sumergiendo a las pajillas en un recipiente con agua temperada 28 a $40{ }^{\circ} \mathrm{C}$ por 40 segundos. Inmediatamente después de la descongelación, se evaluó la motilidad progresiva y la integridad funcional de membrana.

\section{Análisis Estadístico}

El efecto de los tratamientos (momento de adición del glicerol) sobre los porcentajes de motilidad progresiva y porcentajes de integridad funcional de membrana fueron evaluados mediante la prueba de análisis de varianza (ANOVA) y la prueba de Tukey para determinar diferencias entre tratamientos. Se empleó el programa estadístico Prism® v. 3.0.

\section{Resultados}

Los valores mostrados en el Cuadro 1 corresponden a la evaluación inicial de los eyaculados, y se usaron como referentes en el análisis estadístico posterior. 
El efecto de los tratamientos sobre la motilidad progresiva e integridad funcional de membrana después del proceso de congelamiento/descongelamiento se muestran en el Cuadro 2. No se encontró diferencias estadísticas entre tratamientos.

\section{Discusión}

Estudios realizados en otras especies han descrito que el glicerol tiene un efecto nocivo sobre la calidad espermática cuando es añadido al inicio de la curva de enfriamiento, recomendando la adición de glicerol al final de la curva de enfriamiento (sistema de congelamiento en dos pasos) (Foote, 1982; Fiser y Fairfull, 1989; McLaughin et al., 1992; Curry 2000; Holt, 2000). Sin embargo, es posible que el efecto del glicerol a temperaturas entre 30 y $35^{\circ} \mathrm{C}$ para espermatozoides caninos no sea tan marcado.

Se describen métodos de congelamiento en dos pasos, pero adicionando una fracción del glicerol a temperaturas por encima de los $30^{\circ} \mathrm{C}$ (primera fracción del dilutor) y el resto a los $5^{\circ} \mathrm{C}$ en la segunda fracción del dilutor (Peña et al., 1998; Yildtz et al., 2000; Nothling y Shuttleworth, 2005; Silva et al., 2006). En estos estudios se empleó los dos pasos para prevenir o reducir el estrés osmótico que se produciría al exponer el espermatozoide al glicerol en temperaturas mayores a $30^{\circ} \mathrm{C}$ que se emplean en el método de congelamiento de un solo paso.

Los resultados obtenidos al evaluar la motilidad espermática concuerdan con otros estudios (Silva et al., 2003), donde se evaluó el efecto de añadir el semen en uno o dos pasos sobre la motilidad espermática. Sin embargo, en el presente estudio, se evaluó, además, la integridad funcional de membrana; parámetro que también es afectado por el proceso de congelación/descongelación. Existe el consenso que, si bien, el glicerol protege las membranas del espermatozoide durante la criopreservación, también tiene cier- to efecto tóxico sobre ellas (Fahy et al., 1990), pues es probable que el glicerol influya en la reorganización de los fosfolípidos y de las proteínas de la membrana plasmática, afectando así al potencial de fusión de la membrana (Amann y Pickett, 1987; Hammerstedt et al., 1990). Las alteraciones resultantes de este efecto tóxico pueden mermar la fecundidad del espermatozoide, pues aunque presente motilidad tras la descongelación y una adecuada producción de energía, podría estar afectada la capacidad de adhesión al ovocito (Watson, 1979; Amann y Pickett, 1987). Sin embargo, la toxicidad del glicerol afecta en distintos grados a las membranas del espermatozoide, donde parece que las membranas de las mitocondrias son más afectadas que las membranas acrosomale s y plasmáticas (Garner et al., 1999).

En el presente estudio, los resultados muestran valores similares para los parámetros de motilidad e integridad funcional de membrana luego del proceso de descongelamiento en cada uno de los tres tratamientos, indicando que la adición del glicerol al inicio de la curva de enfriamiento ( 35 $\left.{ }^{\circ} \mathrm{C}\right)$ no afectó los parámetros de función espermática. La similitud en los resultados obtenidos entre los tres tratamientos puede deberse a la mayor resistencia del espermatozoide canino, frente a otras especies, con respecto al estrés osmótico y toxicidad ocasionada por el glicerol al ser añadido al inicio de la curva de enfriamiento. Estos resultados son similares al reporte de Silva et al. (2003) en semen canino, donde la adición del total de glicerol al inicio de la curva de enfriamiento (sistema de congelamiento en un paso) no tenía diferencias con la adición de glicerol en otros momentos. Asimismo, en espermatozoides humanos, también se describe el uso de métodos de congelación de un solo paso (Centola et al., 1992). En resumen, la utilización de un sistema de congelamiento en un paso podría ser utilizada para criopreservar espermatozoides caninos sin afectar su calidad espermática. 
Cuadro 1. Características iniciales de las muestras de semen canino utilizadas en el estudio

\begin{tabular}{clcccc}
\hline Muestra & Donante & $\begin{array}{c}\text { Volumen } \\
(\mathrm{mL})\end{array}$ & $\begin{array}{c}\text { Concentración } \\
\left(\mathrm{x} 10^{6} / \mathrm{mL}\right)\end{array}$ & $\begin{array}{c}\text { Motilidad } \\
(\%)\end{array}$ & $\begin{array}{c}\text { HOS }(+) \\
(\%)\end{array}$ \\
\hline 1 & Coqui & 3.0 & 22 & 98 & 95 \\
2 & Tony & 3.0 & 180 & 95 & 76 \\
3 & Coqui & 3.0 & 23 & 92 & 65 \\
4 & Coqui & 4.0 & 21 & 95 & 62 \\
5 & Coqui & 4.0 & 195 & 95 & 60 \\
6 & Oso & 4.0 & 145 & 98 & 81 \\
7 & Coqui & 3.0 & 105 & 96 & 45 \\
8 & Oso & 3.8 & 170 & 95 & 50 \\
9 & Tony & 3.0 & 90 & 85 & 45 \\
10 & Oso & 4.0 & 125 & 95 & 78 \\
11 & Oso & 3.0 & 185 & 98 & 95 \\
12 & Benjy & 3.0 & 30 & 100 & 91 \\
13 & Tony & 3.5 & 65 & 90 & 79 \\
14 & Oso & 3.5 & 270 & 96 & 76 \\
15 & Coqui & 4.0 & 130 & 95 & 90 \\
\hline Prom \pm d.e. & & $3.5 \pm 0.5$ & $117.1 \pm 75.6$ & $94.8 \pm 3.7$ & $72.5 \pm 17.3$ \\
\hline
\end{tabular}

Cuadro 2. Efecto de la adición de glicerol a diferentes momentos de la fase de enfriamiento sobre la motilidad progresiva e integridad funcional de membrana (HOS +) luego del proceso de congelamiento/descongelamiento (valores en porcentaje)

\begin{tabular}{lccc}
\hline & \multicolumn{3}{c}{ Adición de glicerol durante la curva de enfriamiento } \\
\cline { 2 - 4 } & $\mathrm{T} 1-\mathrm{Al}$ inicio & $\mathrm{T} 2-\mathrm{Al}$ inicio y final & $\mathrm{T} 3-\mathrm{Al}$ final \\
\hline Motilidad & $44.4 \pm 7.1$ & $37.1 \pm 12.0$ & $38.4 \pm 8.6$ \\
HOS $(+)$ & $46.5 \pm 23.6$ & $36.4 \pm 20.1$ & $37.9 \pm 16.6$ \\
\hline
\end{tabular}

Los resultados de integridad funcional de membrana ( 38 a $46 \%$ ) fueron ligeramente inferiores a los obtenidos por Sánchez et al. (2002) (53.7 $\pm 13 \%)$. Por otro lado, la motilidad obtenida en el semen descongelado permitiría utilizar el semen en inseminación artificial (Concannon y Battista, 1989).
Los resultados son de gran importancia debido a que el método de congelación de un paso tiene ventajas prácticas, pues minimiza el manejo de la muestra, eliminando un paso durante el proceso de criopreservación y, consecuentemente, reduce las posibilidades de contaminación del semen. 


\section{Conclusiones}

? El momento de adición de glicerol durante la curva de enfriamiento de semen canino no influye sobre la motilidad espermática e integridad funcional de membrana luego del proceso de congelamiento/descongelamiento.

? La adición del glicerol únicamente al inicio de la curva de enfriamiento (método de congelamiento en un paso) puede utilizarse para simplificar la técnica de criopreservación del semen canino.

\section{Literatura Citada}

1. Amann R, Pickett B. 1987. Principles of cryopreservation and a review of cryopreservation of stallion spermatozoa. Equine Vet Sci 7: 145-176.

2. Ax R, Dally M, Didion B, Lenz R, Love C, Varner D, Hafez B, Bellin M. 2000. Semen evaluation. In: Hafez ESE, Hafez B (eds). Reproduction in farm animals. $7^{\mathrm{h}}$ ed. Baltimore: Lippincott Williams \& Wilkins. 509 p.

3. Bateman H. 2001. Effects of semen extender composition and cooling methods on canine sperm function and cryo-survival. MSc Thesis. Ottawa, Canada: Guelph University. 102 p.

4. Centola G, Raubertas R, Mattox J. 1992. Cryopreservation of human semen comparison of cryopreservatives, sources of variability, and prediction of post-thaw survival. J Andrology 13: 283288.

5. Concannon P, Battista M. 1989. Canine semen freezing and artificial insemination. In: Kirk RW. Current veterinary therapy. X. Small animal practice. $10^{\text {th }}$ ed. Philadelphia: WB Saunders. $1421 \mathrm{p}$.

6. Curry M. 2000. Cryopreservation of semen from domestic livestock. Rev Reprod 5: 46-52.

7. England G. 1993. Cryopreservation of dog semen: A review. J Reprod Fert 47: 243-255.
8. Fahy $G$, Lilley $T$, Linsdell $H$, Meryman H. 1990. Cryoprotectant toxicity and cryoprotectant toxicity reduction: in search of molecular mechanisms. Cryobiology 27: 247-268.

9. Fiser P, Fairfull R. 1989. The effect of glycerol-related osmotic changes on post thaw motility and acrosomal integrity of ram spermatozoa. Cryobiology 26: 64-69.

10. Foote R. 1982. Cryopreservation of spermatozoa and artificial insemination: past, present and future. J Androl 3: 85100.

11. Garner D, Thomas C, Gravance C. 1999. The effect of glycerol on the viability, mitochondrial function and acrosomal integrity of bovine spermatozoa. Reprod Domest Anim 34: 399-404.

12. Hammerstedt R, Graham J, Nolan J. 1990. Cryopreservation of mammalian sperm: what we ask them to survive. J Androl 11: 73- 88.

13. Hermansson U, Forsberg C. 2006. Freezing of stored, chilled dog spermatozoa. Theriogenology 65: 584593.

14. Holt W. 2000. Fundamental aspects of sperm cryobiology: The importance of species and individual differences. Theriogenology 53: 47-58.

15. Jeyendran R, Van Der Ven H, PerezPelaes M, Crabo B, Zaneveld L. 1984. Development of an assay to assess the functional integrity of the human sperm membrane and its relationship to other semen characteristics. J Reprod Fert 70: 219-228.

16. Kutzler M. 2005. Semen collection in the dog. Theriogenology 64: 747-754.

17. Linde-Forsberg C. 1995. Artificial insemination with fresh, chilled extended, and frozen-thawed semen in the dog. Semin Vet Med Surg 10: 48-58.

18. McLaughin E, Ford W, Hull M. 1992. The contribution of the toxicity of a glycerol-egg-yolk-citrate cryopreservative to the decline in human sperm motility during cryopreservation. J Reprod Fert 95: 749-754. 
19. Morton D, Bruce S. 1989. Semen evaluation, criopreservation and factors relevant to the use of frozen semen in dogs. J Reprod Fert 39: 311-316.

20. Nothling J, Shuttleworth R. 2005. The effect of straw size, freezing rate and thawing rate upon post-thaw quality of dog semen. Theriogenology 63: 14691480.

21. Peña A, Barrio F, Quintela L, Herradón P. 1998. Effect of different glycerol treatments on frozen-thawed dog sperm longevity and acrosomal integrity. Theriogenology 50: 163-174.

22. Sánchez A, Rubilar J, Gatica R. 2002. Uso de la prueba hipoosmótica en la evaluación de la fertilidad potencial de semen canino fresco y congelado. Arch Med Vet 34(1): 131-134.

23. Sánchez A, Cartagena A, Berland M. 2006. Comparación del efecto de dos diluyentes sobre la fertilidad potencial de semen canino refrigerado. Rev Inv Vet, Perú 17: 1-7.
24. Silva A, Cardoso R, Uchoa D. 2003. Quality of canine semen submitted to single or fractionated glycerol addition during the freezing process. Theriogenology 59: 821-829.

25. Silva A, Cardoso R, Silva L. 2006. Influence of temperature during glycerol addition and post-thaw dilution on the quality of canine frozen semen. Reprod Domest Anim 41: 74-78.

26. Stornelli M, Arauz M, De La Sota L. 2001. Inseminación artificial con semen fresco, refrigerado y congelado, aplicación y desarrollo en caninos. Analecta Vet 21(1): 58-66.

27. Yildtz C, Kaya A, Aksoy M, Tekeli T. 2000. Influence of sugar supplementation of the extender on motility, viability and acrosomal integrity of dog spermatozoa during freezing. Theriogenology 54: 579-585.

28. Watson P. 1979. The preservation of semen in mammals. In: Finn CA. Oxford reviews of reproductive biology. UK: Oxford University Press. 1496 p. 